\title{
Growth and oxidation of graphene on $\mathrm{Rh}(111) \dagger$
}

\author{
Cite this: Phys. Chem. Chem. Phys., 2013, Karin Gotterbarm, Wei Zhao, Oliver Höfert, Christoph Gleichweit, Christian Papp* \\ 15, 19625 \\ and Hans-Peter Steinrück
}

Received 6th September 2013, Accepted 27th September 2013

DOI: $10.1039 / \mathrm{c} 3 \mathrm{cp} 53802 \mathrm{~h}$

www.rsc.org/pccp

\begin{abstract}
The growth and oxidation of graphene supported on $\mathrm{Rh}(111)$ was studied in situ by high-resolution X-ray photoelectron spectroscopy. By variation of propene pressure and surface temperature the optimum growth conditions were identified, yielding graphene with low defect density. Oxidation of graphene was studied at temperatures between 600 and $1000 \mathrm{~K}$, at an oxygen pressure of $\sim 2 \times 10^{-6}$ mbar. The oxidation follows sigmoidal reaction kinetics. In the beginning, the reaction rate is limited by the number of defects, which represent the active sites for oxygen dissociation. After an induction period, the reaction rate increases and graphene is rapidly removed from the surface by oxidation. For graphene with a high defect density we found that the oxidation is faster. In general, a reduction of the induction period and a faster oxidation occur at higher temperatures.
\end{abstract}

\section{Introduction}

Graphene is an intriguing $2 \mathrm{D}$ material ${ }^{1-3}$ with promising applications, e.g. in microelectronics, ${ }^{4-6}$ sensors, ${ }^{7-12}$ as spin-filtering materials, ${ }^{13,14}$ and as protective coatings. ${ }^{15,16}$ One versatile route to fabricate graphene is chemical vapor deposition (CVD) on metal surfaces. ${ }^{17,18}$ This method is particularly interesting, because the graphene-metal interface is important for many of the proposed applications. The morphology and the electronic structure of graphene on a metal substrate strongly depend on the lattice mismatch and on the specific chemical interactions. ${ }^{17-19}$ Graphene on $\mathrm{Rh}(111)$ is considered as an intermediate case, in between strong and weak coupling. ${ }^{19-22}$ On the $\mathrm{Rh}(111)$ single crystal surface, graphene forms a $(12 \times 11)$ Moiré superstructure with a periodicity of $2.9 \mathrm{~nm}$ due to a lattice mismatch of roughly $10 \% .{ }^{20} \mathrm{In}$ those regions of the supercell, where a carbon ring is centered over a bridge position relative to the Rh substrate atoms, hybridization between graphene and $\mathrm{Rh} 4 \mathrm{~d}$ states occurs, leading to a carbon-Rh distance of less than $2.3 \AA$. For the atop position, the interaction between $\mathrm{Rh}$ and graphene is weak and the carbon-metal distance is more than $3 \AA^{2}{ }^{22}$ The resulting complex corrugation pattern has been described in detail by scanning tunneling microscopy (STM) and atomic force microscopy (AFM) measurements. ${ }^{20,21}$

While the structure of graphene on $\mathrm{Rh}(111)$ is well investigated, ${ }^{20-22}$ the growth mechanism during the CVD process on

Lehrstuhl für Physikalische Chemie II, Universität Erlangen-Nürnberg,

Egerlandstraße 3, 91058 Erlangen, Germany. E-mail: christian.papp@fau.de

$\dagger$ Electronic supplementary information (ESI) available: Rh 3d data of the CVD process, a table of the gaussian widths of the graphene signals and the area of the carbidic carbon signal of the discussed graphene layers, measurements of the thermal stability of graphene on $\mathrm{Rh}(111)$ and additional data regarding the oxidation of graphene. See DOI: 10.1039/c3cp53802h metals is still under investigation. Coraux et $a .^{23}{ }^{23}$ underlined the importance of step edges for the nucleation of graphene flakes on $\operatorname{Ir}(111)$, and showed that graphene growth even reshapes the step edges during CVD. Their STM study also indicates that incorporation of carbon adspecies at the graphene edges is the rate limiting step for graphene growth. This finding is supported by electron reflectivity measurements carried out by Loginova et al. on $\operatorname{Ir}(111)$ and also $\operatorname{Ru}(0001) .{ }^{24,25}$ Their results suggest the addition of five-atom carbon clusters rather than single adatoms to the graphene edges as the rate determining step. A rate model based on these data predicts that six of these fiveatom clusters are needed to form a stable nucleus for further graphene growth. ${ }^{26}$ In contrast, on $\mathrm{Rh}(111) \mathrm{STM}$ and density functional theory (DFT) studies indicate $\mathrm{C}_{21}$ and $\mathrm{C}_{24}$ clusters as likely precursors for CVD of graphene. ${ }^{27,28}$ It is suggested that minimizing the number of nucleation sites by controlling the $\mathrm{C}_{21}$ cluster concentration reduces the number of defects in graphene. $^{27}$ Nevertheless, the growth mechanism of graphene on $\mathrm{Rh}(111)$ has not been studied in detail yet. However, the thorough understanding of this mechanism is crucial for the preparation of graphene layers with reproducible quality, i.e. low defect density and large single-crystalline domains. Important parameters to control the quality of graphene are precursor pressure and growth temperature.

Since graphene is also considered as a passivating layer, ${ }^{15,16}$ the stability of graphene in an oxygen atmosphere presents another important issue. The oxidation of graphene layers is also relevant for catalysis, because coke formation can lead to severe site blocking on heterogeneous catalysts. ${ }^{29} \mathrm{On} \mathrm{Ru}(0001)^{30-33}$ and $\operatorname{Ir}(111)^{33-35}$ etching and intercalation of $\mathrm{O}_{2}$ under the graphene layer were observed, depending on the temperature regime. While Cui et al. ${ }^{31}$ observed a linear time-dependence 
for the oxidation of graphene flakes on $\mathrm{Ru}(0001)$, Starodub et $a l .{ }^{33}$ reported a more complex behavior: first the equilibrium between graphene and carbon adclusters is shifted by depleting the surface of carbon adatoms. Only thereafter, oxygen can intercalate under graphene and directly attack the layer from below. On $\operatorname{Ir}(111)$, the oxidation mechanism appears to be completely dominated by intercalation. ${ }^{33}$ Graphene on $\mathrm{Rh}(111)$ is considered as an intermediate system between $\mathrm{Ru}(0001)$ (strong graphene-metal interaction) and $\operatorname{Ir}(111)$ (weak graphene-metal interaction). ${ }^{21}$ One therefore could expect that oxidation of graphene on $\mathrm{Rh}(111)$ follows an intermediate mechanism between those two cases. It should also be noted that oxygen intercalation under graphene has only been observed for coverages below a full monolayer of graphene $e^{30,32-35}$ or oxygen pressures higher than $5 \times 10^{-4}$ mbar. $^{35}$

In this study, we present the in situ investigation of graphene growth on Rh(111) by high-resolution X-ray photoelectron spectroscopy (HRXPS). We systematically explore the influence of precursor pressure and growth temperature on the quality of graphene and derive optimized preparation parameters for graphene growth. As a subsequent step, HRXPS was also employed to monitor the oxidation of graphene monolayers on $\mathrm{Rh}(111)$ at different temperatures.

\section{Experimental details}

All measurements were carried out at beamline U49/2-PGM1 of Helmholtz-Zentrum Berlin in a transportable UHV apparatus, described in more detail elsewhere. ${ }^{36}$ The analyzer chamber is equipped with a three-stage supersonic molecular beam, enabling us to create pressures of up to $10^{-5}$ mbar on the sample while acquiring XP-spectra. The sample was a round $\mathrm{Rh}(111)$ single crystal with $10 \mathrm{~mm}$ diameter and $3 \mathrm{~mm}$ thickness (MaTeck). Temperatures between 80 and $1300 \mathrm{~K}$ are reached by liquid nitrogen cooling in combination with resistive heating. A type $\mathrm{K}$ thermocouple spotwelded to the Rh crystal is used to monitor the sample temperature. The sample was cleaned by Ar sputtering and subsequent annealing to $1100 \mathrm{~K}$. Carbon contaminations (including graphene) were removed by oxidation with $2 \times 10^{-6}$ mbar oxygen at $900 \mathrm{~K}$ and subsequent flashing to $1200 \mathrm{~K}$ to desorb oxygen. Final cleanliness was checked by XPS.

Graphene layers were prepared by CVD of propene at temperatures between 700 and $1000 \mathrm{~K}$ using the molecular beam (to hit the sample directly) or using a background pressure of $\sim 2 \times 10^{-8} \mathrm{mbar}$ in the chamber. Note that when propene was dosed by backfilling the chamber the background pressure in the chamber slowly increased from $8 \times 10^{-9}$ to $2 \times 10^{-8} \mathrm{mbar}$ during the experiment. C 1s spectra were collected during graphene growth until saturation of the C 1s signal was reached. The quality of the graphene layers was verified by low energy electron diffraction (LEED). ${ }^{19,21}$ Additionally we checked the electronic structure of the different graphene layers by measuring the valence band along the $\Gamma$-point $(h \nu=$ $100 \mathrm{eV}$ ) and excellent agreement with earlier results was found for all cases. ${ }^{37}$ Oxygen was dosed directly with the molecular beam creating pressures between $1 \times 10^{-6}$ and $2 \times 10^{-6} \mathrm{mbar}$ on the sample.

C 1s spectra were collected at a photon energy of $380 \mathrm{eV}$, with a total energy resolution of $170 \mathrm{meV}$. The acquisition time was $9 \mathrm{~s}$ per spectrum. All XP spectra were recorded at normal emission. The size of the photon beam on the sample was $125 \mu \mathrm{m}$. Binding energies are referenced to the Fermi level. For the background correction a straight line was subtracted from each spectrum. The components of the C 1s spectra were fitted with asymmetric Doniach-Sunjic functions convoluted with a Gaussian function. ${ }^{38}$ The Lorentzian width for both $\mathrm{C} 1 \mathrm{~s}$ graphene components was kept constant at $0.1 \mathrm{eV}$. However, the Gaussian width varied from 0.23 to $0.68 \mathrm{eV}$ depending on the defect density of the graphene layer and the temperature at which the spectra were recorded.

\section{Results and discussion}

Graphene was grown on the Rh(111) surface by CVD of propene at $\sim 2 \times 10^{-8}$ mbar at elevated temperatures. Selected C $1 \mathrm{~s}$ spectra recorded during the growth process at $920 \mathrm{~K}$ are shown in Fig. 1a. During propene exposure two signals at 285.2 and $284.5 \mathrm{eV}$ arise simultaneously. This behavior is attributed to the corrugation of the graphene sheet on the $\mathrm{Rh}(111)$ surface, which results in carbon atoms with different bonding strengths to the surface. ${ }^{21,22}$ The different surface-graphene bonds lead to two separate signals in the $\mathrm{C} 1 \mathrm{~s}$ region. ${ }^{19}$ The peak at $285.2 \mathrm{eV}$ has a similar binding energy as found for graphene that is strongly coupled to a substrate (e.g. graphene on $\left.\mathrm{Ni}(111)^{39}\right)$. This contribution (referred to as $C_{1}$ ) is therefore assigned to carbon atoms in the Moire unit cell, which bond strongly to the surface, i.e. where a carbon ring is centered over a bridge position. The binding energy of $284.5 \mathrm{eV}$ is close to the value found for weakly coupled or quasi-freestanding graphene. ${ }^{19}$ Hence this contribution (referred to as $C_{2}$ ) is assigned to carbon atoms further away from the surface. A typical fit is shown in Fig. 1b. The Lorentzian width is $100 \mathrm{meV}$ for both contributions and the Gaussian widths are 410 and $360 \mathrm{meV}$ for $C_{1}$ and $C_{2}$, respectively. Fig. 1c shows the fit results for the data shown in Fig. 1a. The growth rate is constant in the beginning and slows down with increasing graphene coverage. This behavior is attributed to the decrease of uncovered $\mathrm{Rh}$ surface regions available for propene adsorption and subsequent dehydrogenation. At $\sim 30$ Langmuir $(\mathrm{L})$, the graphene coverage reaches saturation. The $C_{1}$ signal is around 1.4 times higher than the $C_{2}$ signal, which indicates (neglecting photoelectron diffraction effects) a larger fraction of strongly bound graphene atoms during the entire growth process. Rh $3 \mathrm{~d}$ spectra recorded during the growth process are available in the ESI, $\uparrow$ Fig. S1.

After graphene growth the sample is cooled down and C 1s spectra are recorded during the cooling process. Selected spectra are shown in Fig. 2a. During cooling from 925 to $155 \mathrm{~K}$, the peak at $284.5 \mathrm{eV}$ increases in relative intensity. Additionally both contributions shift further apart by $50 \mathrm{meV}$ and decrease in width. Fig. 2b shows that the Gaussian widths of both contributions decrease with temperature (by $58 \mathrm{meV}$ for $C_{1}$ and $97 \mathrm{meV}$ for $C_{2}$ ). 
a)
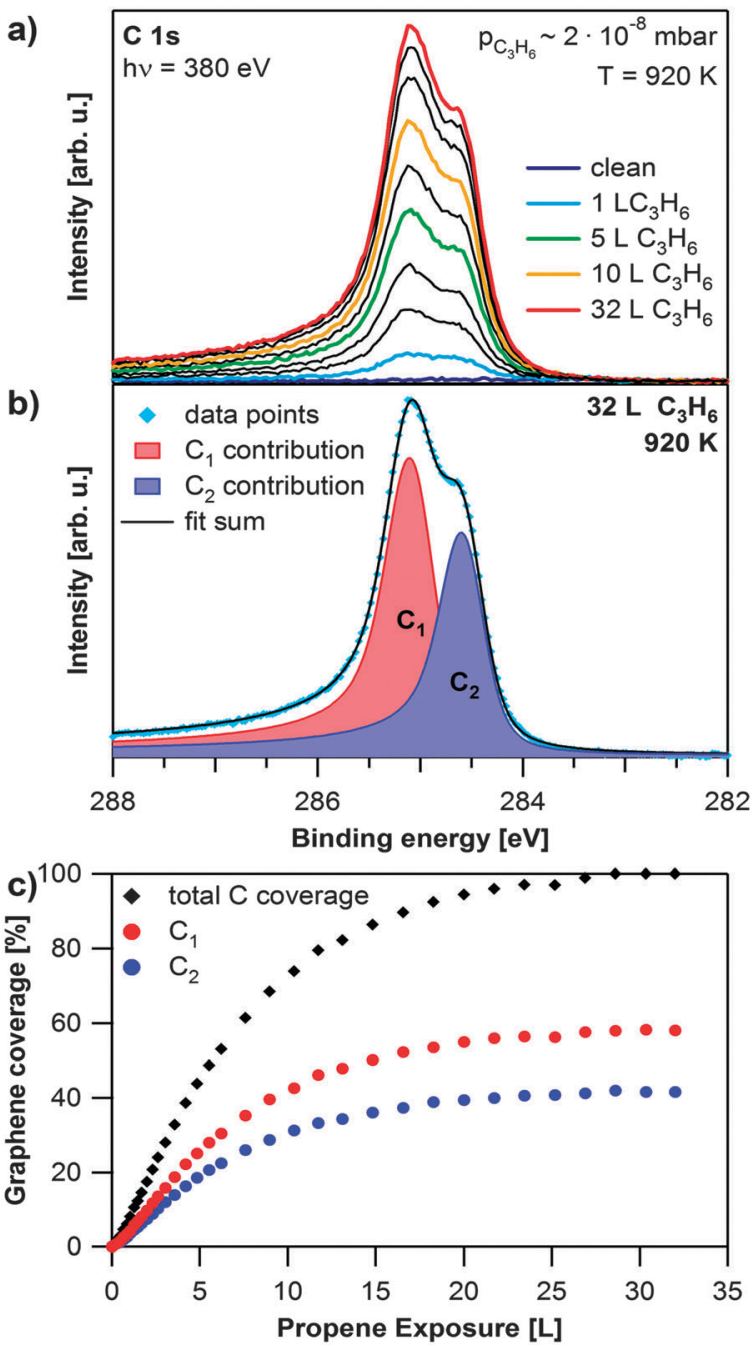

Fig. 1 Growth of graphene on $\mathrm{Rh}(111)$. (a) Selected C 1s spectra during propene exposure at $920 \mathrm{~K}$; the propene exposure of the coloured spectra is shown in the legend, black spectra are recorded at exposures in between the given values; during the experiment the pressure in the chamber slowly increased from $8 \times 10^{-9}$ to $2 \times 10^{-8}$ mbar. (b) Fit of saturated $C 1 \mathrm{~s}$ spectrum. (c) Quantitative analysis of the experiment shown in (a).

We attribute this decrease to the fact that at lower temperatures less vibrations of the graphene layer are excited and thus the local environment is more defined, leading to a smaller linewidth. This effect is less pronounced for the more strongly bound carbon atoms, and thus the narrowing of the $C_{1}$ signal is smaller than for the $C_{2}$ signal. During cooling, the ratio between the two carbon species decreases from 1.4 to 1.2 indicating that at lower temperatures more carbon atoms are in the weakly coupled state with a larger distance from the Rh surface. In order to verify that we obtained a uniform graphene layer, we measured C 1s spectra at $155 \mathrm{~K}$ over the whole sample; except for regions close to the edge of the crystal, identical spectra were observed. This is in line with our LEED measurements (data not shown), which always showed a clear Moiré pattern. ${ }^{19,21}$

To elucidate the influence of the growth parameters for graphene on $\mathrm{Rh}(111)$, both propene pressure and sample a)
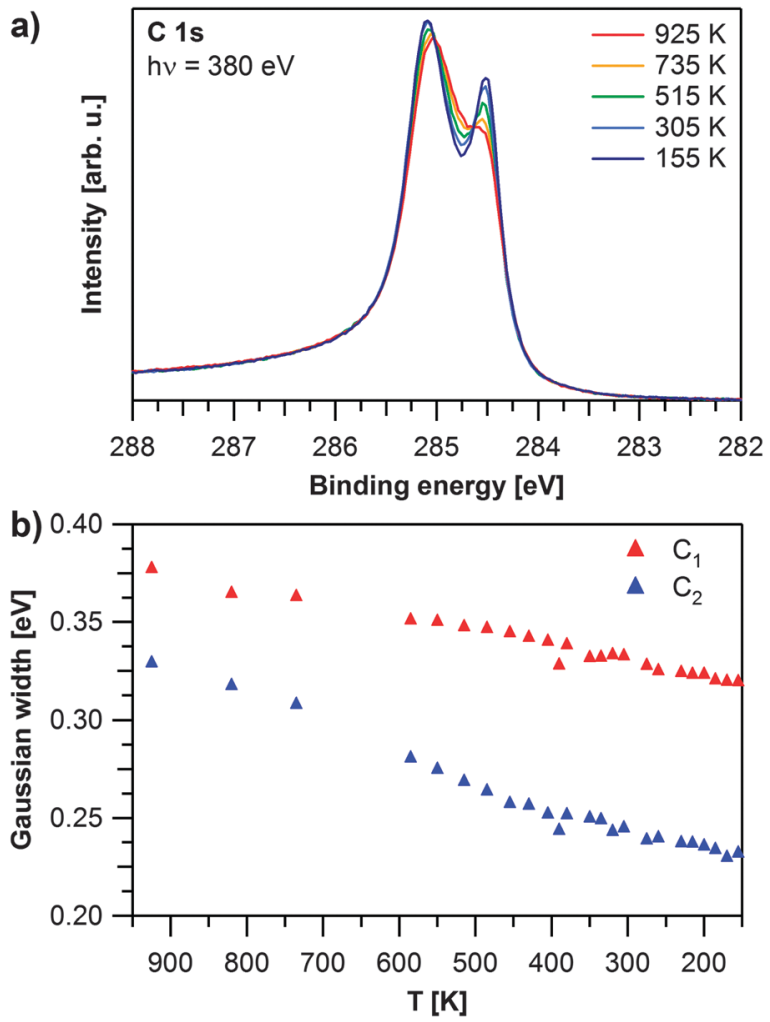

Fig. 2 Temperature dependent evolution of graphene on $\mathrm{Rh}(111)$ : (a) selected $C$ 1s spectra recorded during cooling down. (b) Gaussian widths of the C 1s contributions as obtained by fitting the experiment shown in (a).

temperature were varied, yielding graphene layers of different quality. The spectra of graphene layers grown at temperatures from 700 to $1000 \mathrm{~K}$, at propene pressures of $2 \times 10^{-8}$ or $2 \times 10^{-6}$ mbar are depicted in Fig. 3 . They were recorded below $150 \mathrm{~K}$ to reduce the influence of vibrations in the graphene layer.

First we discuss the influence of the precursor pressure on graphene growth. Fig. 3c shows the spectrum of a graphene layer grown at $920 \mathrm{~K}$ at $2 \times 10^{-8}$ mbar. Graphene grows slowly and a closed layer is reached after $45 \mathrm{~min}$ (32 L) (see also Fig. 1). The layer shown in Fig. 3e was grown at the same temperature, but at a pressure of $2 \times 10^{-6}$ mbar on the sample (achieved with the molecular beam hitting the sample directly), and is saturated already after $40 \mathrm{~s}(80 \mathrm{~L})$. For the higher pressure and shorter preparation time, the $\mathrm{C} 1 \mathrm{~s}$ graphene peaks are broad and not clearly separated. The Gaussian width is $0.54 \mathrm{eV}$ for the $C_{1}$ contribution at $285.2 \mathrm{eV}$ and $0.66 \mathrm{eV}$ for the $C_{2}$ shoulder at $284.6 \mathrm{eV}$. A third carbon contribution from carbidic carbon forms at $283.8 \mathrm{eV}$. LEED measurements of this graphene layer showed a diffuse circle around the substrate spots instead of the clearly resolved Moiré pattern observed before, indicating multiple rotational graphene domains. From the comparison of the $\mathrm{C} \mathrm{1s}$ spectra of the two layers prepared at $920 \mathrm{~K}$ but with different precursor pressures, we conclude that the width of the $C_{1}$ and $C_{2}$ peaks is an indicator of the quality (i.e. defect density) of the graphene layers. The Gaussian widths and carbidic carbon content for all spectra displayed in Fig. 3 are 


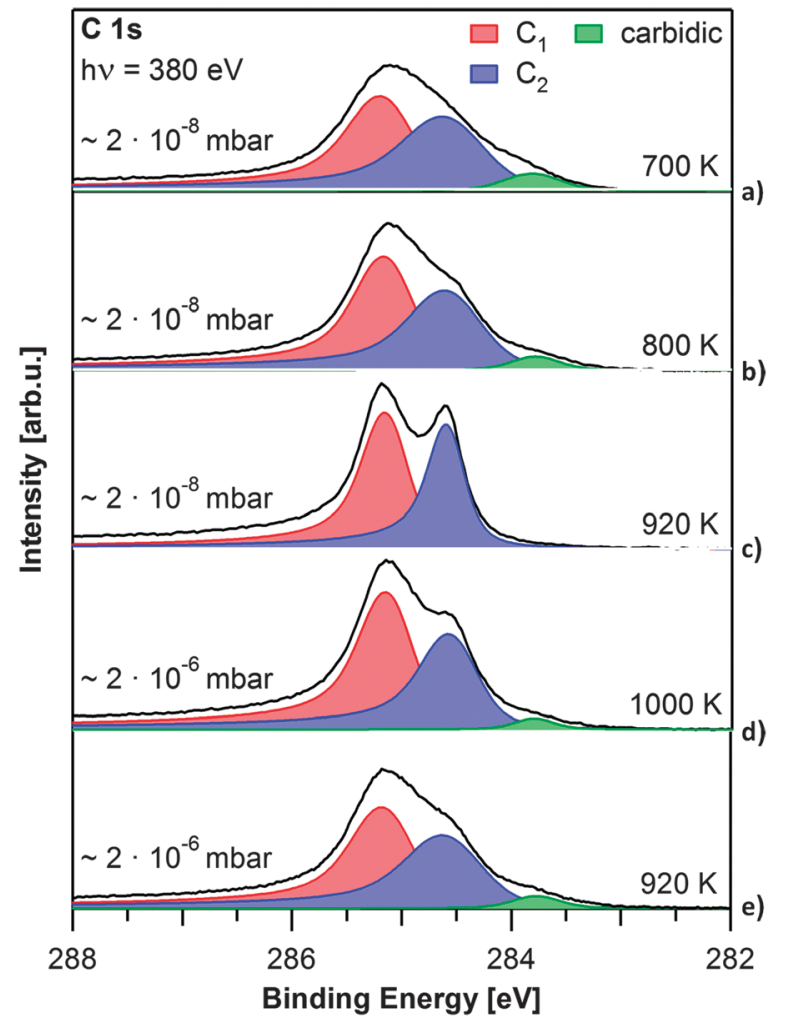

Fig. 3 C 1s spectra of graphene layers grown at different pressures and temperatures, as denoted. All spectra were recorded at $T<150 \mathrm{~K}$.

listed in Table S2 in the ESI. $†$ The higher defect density found for the higher precursor pressure can be explained as follows: at higher precursor pressure the carbon adatom concentration is well above the concentration necessary to nucleate graphene islands. ${ }^{24-26}$ Therefore nucleation simultaneously occurs at numerous sites, leading to a large number of small graphene domains and consequently domain boundaries, yielding a high defect density in the resulting layer. From our measurements we conclude that lower precursor pressures lead to graphene layers with a lower defect density.

Next, we discuss the influence of the substrate temperature: Fig. 3a-c show spectra of graphene layers prepared at the lower propene pressure of $2 \times 10^{-8} \mathrm{mbar}$ at 700,800 and $920 \mathrm{~K}$, respectively. The layer grown at $700 \mathrm{~K}$ exhibits broad $\mathrm{C} 1 \mathrm{~s}$ signals (Gaussian widths: 0.59 and $0.68 \mathrm{eV}$ for $C_{1}$ and $C_{2}$, respectively), and a carbidic carbon signal at $283.8 \mathrm{eV}$. With increasing temperature, the graphene signals become more distinct and the carbidic carbon contribution at $283.8 \mathrm{eV}$ decreases $(800 \mathrm{~K})$ or vanishes completely $(920 \mathrm{~K})$ (see also Table S2, ESI $\dagger$ ). This temperature dependence can be explained considering the results of Yuan et al. ${ }^{27}$ They found that the lifetime of $\mathrm{C}_{21}$ clusters, which are likely to be the nucleation sites for graphene growth, decreases with temperature. It is suggested that minimizing the number of nucleation sites will lead to less defects in the resulting graphene layer. At even higher temperatures carbon starts to dissolve into the bulk of the crystal and carbidic carbon from the subsurface can diffuse to the surface. From thermal stability experiments (see ESI, $\uparrow$ Fig. S3) we conclude that the onset of carbon diffusion into the bulk of the crystal lies between 1000 and $1100 \mathrm{~K}$. Therefore, we were not able to grow graphene at $1000 \mathrm{~K}$ using $2 \times 10^{-8}$ mbar propene, but had to increase the propene pressure to $2 \times 10^{-6} \mathrm{mbar}$. The corresponding C 1s spectrum in Fig. 3d shows significantly broader peaks than the one for growth at $920 \mathrm{~K}$, along with a small carbidic carbon contribution at $283.8 \mathrm{eV}$. The Gaussian widths are $0.51 \mathrm{eV}$ for the $C_{1}$ peak and $0.53 \mathrm{eV}$ for the $C_{2}$ peak. Due to the higher propene pressure, saturation is again reached faster, leading to more domains and therefore a higher defect density (see discussion of Fig. 3e). To conclude this section, our pressure- and temperature-dependent measurements show that for graphene growth on $\mathrm{Rh}(111)$, a temperature of $920 \mathrm{~K}$ and a propene pressure of $2 \times 10^{-8}$ mbar represent the best conditions to produce layers with extended domains and few defects.

As next topic, we discuss the oxidation of the saturated graphene layer, grown at $920 \mathrm{~K}$ and a propene pressure of $2 \times 10^{-8}$ mbar, see also Fig. 3c. Selected spectra recorded during the oxidation of graphene with $1 \times 10^{-6}$ mbar oxygen at $900 \mathrm{~K}$ are shown in Fig. 4a. After an induction period of $\sim 150 \mathrm{~L}$ $(200 \mathrm{~s})$, both carbon species start to vanish simultaneously. The carbon coverage continues to decrease, until no carbon is left on the surface after an exposure of $633 \mathrm{~L}$ of oxygen (red spectrum). Since we do not observe any other carbon species and carbon-oxygen groups we conclude that graphene is etched

a)
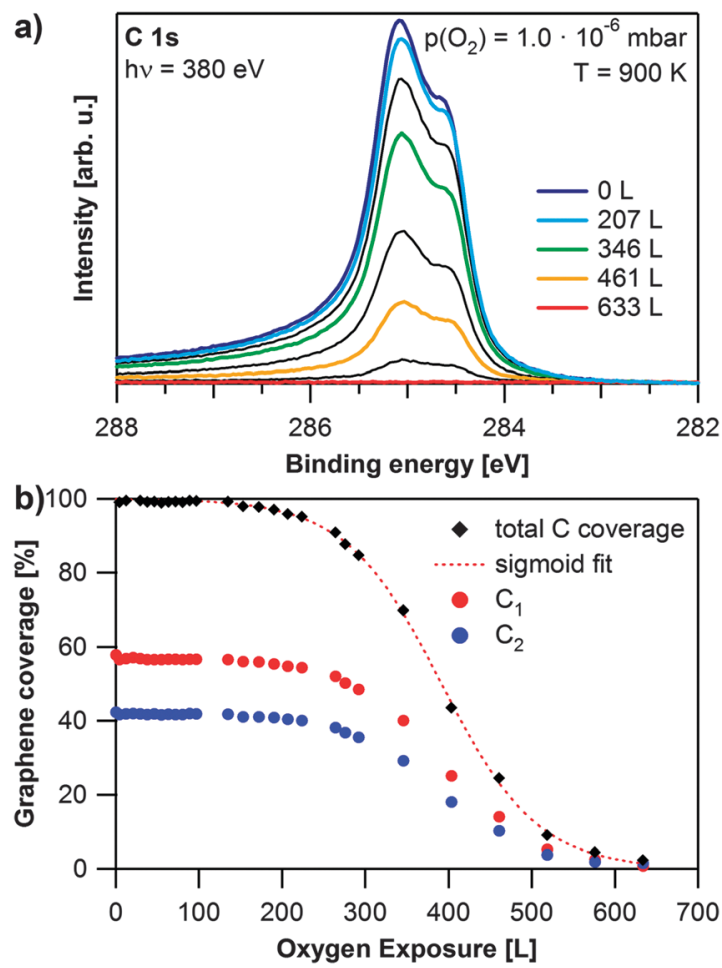

Fig. 4 Oxidation of graphene on $\mathrm{Rh}(111)$ : (a) selected spectra during oxidation with $1 \times 10^{-6}$ mbar $_{2}$ at $900 \mathrm{~K}$, the oxygen exposure of the coloured spectra is shown in the legend, black spectra are recorded at exposures in between the given values; (b) quantitative analysis of the experiment shown in (a) as obtained from fitting, red dotted line: sigmoidal fit of the total graphene coverage. 
by exposure to oxygen and directly desorbed as CO or possibly $\mathrm{CO}_{2}$. The quantitative analysis of this experiment, shown in Fig. $4 \mathrm{~b}$, reveals S-shaped reaction kinetics. The first part of the reaction is an induction period, in which the carbon coverage stays almost constant. The induction period lasts until an exposure of approximately $150 \mathrm{~L}$ of oxygen. Thereafter, the carbon coverage decreases quickly until $450 \mathrm{~L}$. The last part of the reaction resembles an exponential decay, which would be typical for a first or pseudo-first order reaction.

The observed behavior can be explained, if we consider the relation between the number of free adsorption sites and the carbon coverage. Initially, the whole rhodium surface is covered with graphene. Therefore oxygen can only adsorb and dissociate at defects in the graphene layer. For $\operatorname{Ir}(111)$, graphene oxidation has, e.g. been reported to start at wrinkles of the graphene layer. ${ }^{33}$ In this initial regime, the reaction rate is limited by the oxygen coverage (i.e., the number of free adsorption sites). After the induction period, more and more adsorption sites on the $\mathrm{Rh}$ surface become available and the reaction rate increases. Subsequently, the (decreasing) carbon coverage becomes the rate-limiting factor causing the reaction rate to slow down again. Since oxygen is supplied in excess from the gas phase, the reaction rate depends only on the carbon coverage at this stage. Therefore the reaction can be considered as a pseudo first order reaction in this regime. The observed profile can be described by a sigmoidal curve as shown by the fit (dotted red line). At temperatures below $600 \mathrm{~K}$ (not shown), we observed no change in the $\mathrm{C} 1 \mathrm{~s}$ signal, even after exposure to $1000 \mathrm{~L}$ of oxygen. This is in contrast to previous studies of graphene on $\mathrm{Ru}(0001)$ and $\operatorname{Ir}(111)$, which reported intercalation of oxygen below $600 \mathrm{~K} .^{30,34,35}$ Since we did not observe any signal in the $\mathrm{O}$ 1s region, intercalation of oxygen under the saturated graphene layer does not occur under the conditions applied in our study. Furthermore, even after exposure of a graphene layer to air for four hours we found no significant changes in the $\mathrm{C} 1 \mathrm{~s}$ and Rh 3d core levels.

To elucidate the influence of defects, we studied the oxidation of two different saturated graphene layers on $\mathrm{Rh}(111)$, which were grown at $920 \mathrm{~K}$ at different propene pressures. Fast grown layers $\left(2 \times 10^{-6}\right.$ mbar propene, Fig. 3e) are referred to as high defect density graphene, slowly grown layers $\left(2 \times 10^{-8} \mathrm{mbar}\right.$ propene, Fig. 3c) are referred to as low defect density graphene. For both layers, we investigated the oxidation at $2 \times 10^{-6} \mathrm{mbar}$ of oxygen in the temperature range from 600 to $1000 \mathrm{~K}$. The corresponding results are shown in Fig. 5. The data points correspond to the total graphene coverage. All experiments show the same S-shaped time dependent behavior and can be fitted with sigmoidal curves (dotted lines).

Fig. 5a shows a series of oxidation experiments with low defect density graphene, carried out at temperatures between 700 and $850 \mathrm{~K}$. The length of the induction period decreases and the reaction rate increases with temperature. Note that the data in Fig. $4 \mathrm{~b}$ at $900 \mathrm{~K}$ show a somewhat longer induction period than the data at $850 \mathrm{~K}$ in Fig. $5 \mathrm{a}$ (see also Fig. $\mathrm{S} 4$ in the ESI $\dagger$ ). This behavior does not fit into the overall trend and seems to be a discrepancy at first glance. It can, however,

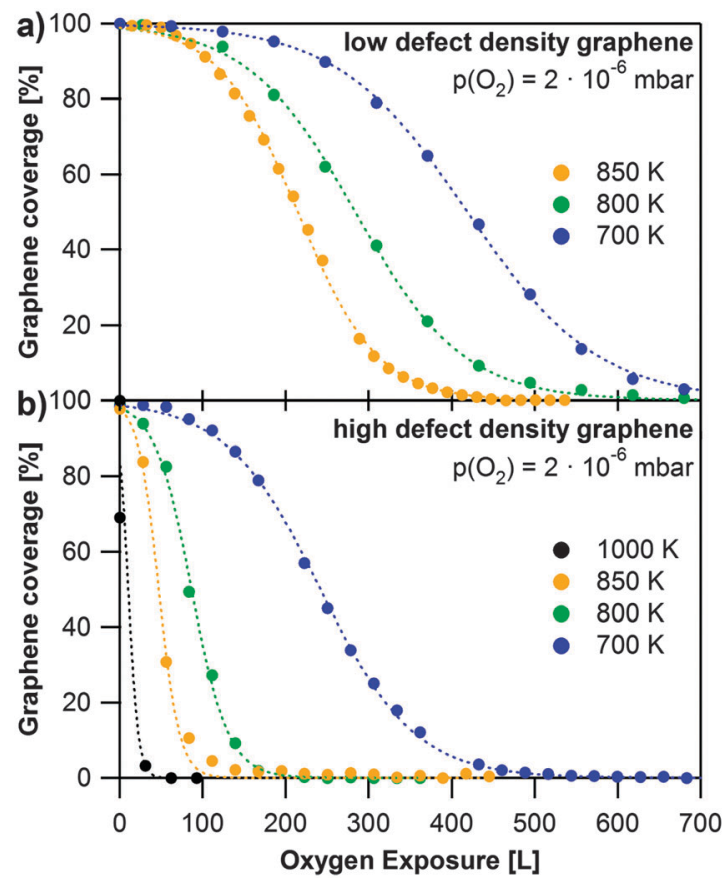

Fig. 5 Oxidation of graphene at different temperatures with $2 \times 10^{-6} \mathrm{mbar}$ oxygen. The data points are the sum of both graphene $C 1 \mathrm{~s}$ contributions, dotted lines display sigmoidal fits of the data. (a) Oxidation of low defect density graphene; (b) oxidation of high defect density graphene.

be understood, if one considers that layers, which have been prepared independently under nominally the same conditions, can display different defect densities. While this influences the length of the induction period, it should not influence the slope of the subsequent decrease due to oxidation, which indeed occurs faster with increasing temperatures for all layers studied - see Fig. 5a (and Fig. S4 in the ESI $\dagger$ ).

The series of oxidation experiments with high defect density graphene is displayed in Fig. 5b. In comparison to the data shown in Fig. 5a it is obvious that graphene sheets with a higher defect density are oxidized much faster than low defect density sheets under the same oxidation conditions. Especially the induction period is much shorter, since the oxidation starts at defects in the layer. The reaction rate again increases with temperature, as expected. It is interesting to note that for high defect density graphene we also observed etching at $600 \mathrm{~K}$. However, at this temperature oxidation is very slow with an induction period of roughly $1000 \mathrm{~L}(1500 \mathrm{~s})$. The layer was completely oxidized after exposure to $4000 \mathrm{~L}$ of oxygen.

Our data clearly show the great influence of the defect density on the oxidation rate. Ideally, one would aim at deriving the detailed reaction kinetics from the data in Fig. 5. We however, refrain from such an analysis due to the following reasons: XPS is a technique integrating over a large surface area as compared to the typical size of a graphene domain. Nevertheless regarding the high quality of the graphene layer the number of domain boundaries (and thus defects) and also the size of the domains probed will vary. The measured overall reaction rate, as deduced from Fig. 5, will be the sum of 
different induction periods and also different domain sizedependent oxidation rates. We thus have to conclude that while our measurements clearly allow demonstrating a dramatic decrease of the induction time and increase of the reaction rate, they do not permit us to determine detailed kinetic parameters such as the activation energy. Here, ideally local probes could be beneficial, which however face inherent problems in the in situ quantitative analysis at elevated temperatures.

\section{Conclusions}

We investigated the growth and oxidation of graphene on $\mathrm{Rh}(111)$ by in situ HRXPS. In agreement with previous studies, we find two C 1s species indicative of graphene regions exhibiting strong and weak interactions with the Rh substrate. The width of the $\mathrm{C} 1 \mathrm{~s}$ signals is considerably temperature dependent due to vibrations of the graphene layer. By systematically exploring the preparation parameters we were able to optimize the graphene growth conditions. CVD of graphene on $\mathrm{Rh}(111)$ at $920 \mathrm{~K}$ and $2 \times 10^{-8} \mathrm{mbar}$ propene pressure leads to layers with low defect density and large, single crystalline domains. We present the first report of oxidation of graphene on $\mathrm{Rh}(111)$. Oxidation of graphene was observed at temperatures between 600 and $1000 \mathrm{~K}$ and at an oxygen pressure of $\sim 2 \times 10^{-6}$ mbar. We find that the oxidation follows sigmoidal reaction kinetics. In the beginning the reaction rate is governed by the concentration of oxygen on the surface, which is determined by the number of free adsorption sites. After this induction period, the reaction rate accelerates and graphene is rapidly removed from the surface. The length of the induction period decreases and the rate constant increases with defect density in the graphene layers, and also with surface temperature. The reaction kinetics turned out to be very complex, which is attributed to the fact that both the length of the induction period and the reaction rate depend on the size of the graphene domains and defect density.

\section{Acknowledgements}

The authors gratefully acknowledge the funding from SFB 953 "Synthetic Carbon Allotropes" and the German Research Council (DFG), which, within the framework of its "Excellence Initiative", supports the Cluster of Excellence "Engineering of Advanced Materials" (www.eam.uni-erlangen.de) at the Universität Erlangen-Nürnberg. We thank HZB for the allocation of synchrotron radiation beamtime and the BESSY staff for support during beamtime. K. G. thanks the "Fonds der chemischen Industrie" for providing her a PhD grant. W. Z. thanks the China Scholarship Council for financing his PhD grant.

\section{References}

1 K. S. Novoselov, A. K. Geim, S. V. Morozov, D. Jiang, Y. Zhang, S. V. Dubonos, I. V. Grigorieva and A. A. Firsov, Science, 2004, 306, 666-669.

2 K. S. Novoselov, A. K. Geim, S. V. Morozov, D. Jiang, M. I. Katsnelson, I. V. Grigorieva, S. V. Dubonos and A. A. Firsov, Nature, 2005, 438, 197-200.
3 K. S. Novoselov, Rev. Mod. Phys., 2011, 83, 837-849.

4 Y.-M. Lin, C. Dimitrakopoulos, K. A. Jenkins, D. B. Farmer, H.-Y. Chiu, A. Grill and P. Avouris, Science, 2010, 327, 662.

5 Y.-M. Lin, A. Valdes-Garcia, S.-J. Han, D. B. Farmer, I. Meric, Y. Sun, Y. Wu, C. Dimitrakopoulos, A. Grill, P. Avouris and K. A. Jenkins, Science, 2011, 332, 1294-1297.

6 S. Bae, H. Kim, Y. Lee, X. Xu, J.-S. Park, Y. Zheng, J. Balakrishnan, T. Lei, H. Ri Kim, Y. I. Song, Y.-J. Kim, K. S. Kim, B. Ozyilmaz, J.-H. Ahn, B. H. Hong and S. Iijima, Nat. Nanotechnol., 2010, 5, 574-578.

7 S. Alwarappan, S. Boyapalle, A. Kumar, C.-Z. Li and S. Mohapatra, J. Phys. Chem. C, 2012, 116, 6556-6559.

8 S. Alwarappan, K. Cissell, S. Dixit, C.-Z. Li and S. Mohapatra, J. Electroanal. Chem., 2012, 686, 69-72.

9 S. Alwarappan, A. Erdem, C. Liu and C.-Z. Li, J. Phys. Chem. C, 2009, 113, 8853-8857.

10 S. Alwarappan, C. Liu, A. Kumar and C.-Z. Li, J. Phys. Chem. C, 2010, 114, 12920-12924.

11 S. Alwarappan, S. R. Singh, S. Pillai, A. Kumar and S. Mohapatra, Anal. Lett., 2012, 45, 746-753.

12 F. Schedin, A. K. Geim, S. V. Morozov, E. W. Hill, P. Blake, M. I. Katsnelson and K. S. Novoselov, Nat. Mater., 2007, 6, 652-655.

13 V. M. Karpan, G. Giovannetti, P. A. Khomyakov, M. Talanana, A. A. Starikov, M. Zwierzycki, J. van den Brink, G. Brocks and P. J. Kelly, Phys. Rev. Lett., 2007, 99, 176602.

14 S. D. Yu and M. Fonin, New J. Phys., 2010, 12, 125004.

15 E. Sutter, P. Albrecht, F. E. Camino and P. Sutter, Carbon, 2010, 48, 4414-4420.

16 S. Chen, L. Brown, M. Levendorf, W. Cai, S.-Y. Ju, J. Edgeworth, X. Li, C. W. Magnuson, A. Velamakanni, R. D. Piner, J. Kang, J. Park and R. S. Ruoff, ACS Nano, 2011, 5, 1321-1327.

17 J. Wintterlin and M.-L. Bocquet, Surf. Sci., 2009, 603, 1841-1852.

18 M. Batzill, Surf. Sci. Rep., 2012, 67, 83-115.

19 A. B. Preobrajenski, M. L. Ng, A. S. Vinogradov and N. Martensson, Phys. Rev. B: Condens. Matter Mater. Phys., 2008, 78, 073401.

20 B. Wang, M. Caffio, C. Bromley, H. Früchtl and R. Schaub, ACS Nano, 2010, 4, 5773-5782.

21 E. N. Voloshina, Y. S. Dedkov, S. Torbrügge, A. Thissen and M. Fonin, Appl. Phys. Lett., 2012, 100, 241606.

22 M. Iannuzzi and J. Hutter, Surf. Sci., 2011, 605, 1360-1368. 23 J. Coraux, A. T. N'Diaye, M. Engler, C. Busse, D. Wall, N. Buckanie, F. Meyer zu Heringdorf, R. van Gastel, B. Poelsema and T. Michely, New J. Phys., 2009, 11, 023006.

24 E. Loginova, N. C. Bartelt, P. J. Feibelman and K. F. McCarty, New J. Phys., 2008, 10, 093026.

25 E. Loginova, N. C. Bartelt, P. J. Feibelman and K. F. McCarty, New J. Phys., 2009, 11, 063046.

26 A. Zangwill and D. D. Vvedensky, Nano Lett., 2011, 11, 2092-2095.

27 Q. Yuan, J. Gao, H. Shu, J. Zhao, X. Chen and F. Ding, J. Am. Chem. Soc., 2011, 134, 2970-2975. 
28 B. Wang, X. Ma, M. Caffio, R. Schaub and W.-X. Li, Nano Lett., 2011, 11, 424-430.

29 P. Forzatti and L. Lietti, Catal. Today, 1999, 52, 165-181.

30 P. Sutter, J. T. Sadowski and E. A. Sutter, J. Am. Chem. Soc., 2010, 132, 8175-8179.

31 Y. Cui, Q. Fu, H. Zhang, D. Tan and X. Bao, J. Phys. Chem. C, 2009, 113, 20365-20370.

32 H. Zhang, Q. Fu, Y. Cui, D. Tan and X. Bao, J. Phys. Chem. C, 2009, 113, 8296-8301.

33 E. Starodub, N. C. Bartelt and K. F. McCarty, J. Phys. Chem. C, 2010, 114, 5134-5140.

34 E. Grånäs, J. Knudsen, U. A. Schröder, T. Gerber, C. Busse, M. A. Arman, K. Schulte, J. N. Andersen and T. Michely, ACS Nano, 2012, 6, 9951-9963.
35 R. Larciprete, S. Ulstrup, P. Lacovig, M. Dalmiglio, M. Bianchi, F. Mazzola, L. Hornekær, F. Orlando, A. Baraldi, P. Hofmann and S. Lizzit, ACS Nano, 2012, 6, 9551-9558.

36 R. Denecke, M. Kinne, C. M. Whelan and H.-P. Steinrück, Surf. Rev. Lett., 2002, 9, 797-801.

37 M. Sicot, P. Leicht, A. Zusan, S. Bouvron, O. Zander, M. Weser, Y. S. Dedkov, K. Horn and M. Fonin, ACS Nano, 2012, 6, 151-158.

38 S. Doniach and M. Šunjić, J. Phys. C: Solid State Phys., 1970, 3, 285.

39 W. Zhao, S. M. Kozlov, O. Höfert, K. Gotterbarm, M. P. A. Lorenz, F. Vines, C. Papp, A. Görling and H.-P. Steinrück, J. Phys. Chem. Lett., 2011, 2, 759-764. 